\title{
Evaluation and Optimization of Paper-Based SERS Substrate for Potential Label-Free Raman Analysis of Seminal Plasma
}

\author{
Zufang Huang, ${ }^{1}$ Gang Cao, ${ }^{1}$ Yan Sun, ${ }^{2}$ Shengrong Du, ${ }^{2}$ Yongzeng Li, ${ }^{1}$ \\ Shangyuan Feng, ${ }^{1}$ Juqiang Lin, ${ }^{1}$ and Jinping Lei ${ }^{1}$ \\ ${ }^{1}$ Key Laboratory of Optoelectronic Science and Technology for Medicine of Ministry of Education, \\ Fujian Provincial Key Laboratory of Photonics Technology, Fujian Normal University, Fuzhou 350007, China \\ ${ }^{2}$ Fujian Maternal and Child Health Hospital, Fuzhou 350001, China \\ Correspondence should be addressed to Zufang Huang; zhuang18@jhu.edu
}

Received 16 April 2017; Revised 8 July 2017; Accepted 18 July 2017; Published 28 August 2017

Academic Editor: Rajesh R. Naik

Copyright (c) 2017 Zufang Huang et al. This is an open access article distributed under the Creative Commons Attribution License, which permits unrestricted use, distribution, and reproduction in any medium, provided the original work is properly cited.

\begin{abstract}
Characterization and optimization of paper SERS substrate were performed in detail, in which morphologies and distribution of silver nanoparticles (AgNPs) on the paper substrate pretreated with different concentrations of $\mathrm{NaCl}$ and the subsequent soaking with colloidal AgNPs for different period of time were evaluated. Our results show that both $\mathrm{NaCl}$ concentration and soaking time with AgNPs have a significant influence on SERS enhancement, showing that an optimal EF of $2.27 \times 10^{7}$ was achieved when the paper substrate was treated with $20 \mathrm{mM} \mathrm{NaCl}$ and one-hour soak with AgNPs. Moreover, seminal plasma (SP) was specifically selected to evaluate the performance of paper-based SERS substrate for potential clinical detection and diagnosis. The optimization of the paper SERS substrate demonstrates potential applications in reliable on-site detection of SP and clinical diagnosis of fertilityrelated diseases as well.
\end{abstract}

\section{Introduction}

Compared with conventional Raman spectroscopy, surfaceenhanced Raman spectroscopy (SERS) can provide highsensitivity detection due to its large enhancement to Raman signals [1-3]. It is therefore identified as a potentially powerful technique for biomedical and biochemical analysis in which Raman signals were usually overwhelmed by intense fluorescence background. Typically, SERS-active metal nanostructures (e.g., nanoparticles, nanowires, nanotubes, and nanorods) are fabricated via either chemical or physical methods [4]. It is still quite challenging to obtain colloidal nanoparticles with high uniformity while avoiding uncontrolled aggregations by "wet chemistry syntheses" [5]. By contrast, solid substrates created via electron beam lithography [6] focused ion beam patterning or thermal evaporation method [7] indeed demonstrate great reproducibility; the drawbacks are however the high cost and complicated fabrication required; additionally, some commercially available solid SERS substrates have relatively low enhancement factors.
Therefore, extensive efforts have been devoted to developing high-performance SERS substrates, particularly those that are low in cost and easy to fabricate. Paper-based SERS substrate not only has the advantages of low cost and ease of fabrication [8] but also demonstrates both high-sensitivity and great reproducibility $[9,10]$, making it a facile platform for applications in fields such as forensic science, food safety, and environment protection [11-13]; particularly the unique properties of paper (e.g., flexibility, hydrophilicity) are wellsuited for analysis of samples with arbitrary shapes and trace level as well as those that cannot be easily transferred to lab. Printing nanoparticles directly onto the paper via commercial inkjet printer is a rapid and low-cost method for fabrication of paper-based SERS substrate [12, 14]. However, clogging of nozzles sometimes happens due to the high-viscosity inks used for sufficient loadings; moreover, relatively lower SERS performance was observed compared with soaking method [10]. One explanation is that nanoparticles cannot easily penetrate into the bulk of paper by the printing method, resulting in the substrate not being able to achieve high SERS performance via interlayer enhancement [9]. By 
contrast, paper SERS substrates prepared through soaking the filter paper with colloidal nanoparticles demonstrate higher enhancement factors of up to $10^{8}$ [10]. Previous studies showed that to achieve high SERS performance, the soaking time mostly took one to two days [15], which may cause potential oxidation to Ag nanoparticles during the process as well as the potential decay of the filter paper. Therefore, a new paradigm to enable high-performance and rapid fabrication of paper SERS substrate is needed.

For optimization of SERS substrates, halide ions are simple and useful chemicals used to induce the aggregation of colloidal nanoparticles to create more SERS-active "hot spots," which give rise to large surface plasmon resonances to yield higher SERS enhancement during measurement [16]. $\mathrm{NaCl}$ was reported as one of the most effective aggregating agents that can be used prior to the soaking of filter paper with concentrated colloidal silver nanoparticles (AgNPs) $[17,18]$. The aggregated metallic nanoparticles yield great signal enhancement; however, the orders of addition and the mixing ratio of nanoparticles and aggregation agent have a crucial influence on the SERS signal. Insufficient chloride ions would not overcome electrostatic repulsion between AgNPs effectively to achieve more hotspots; while excessive chloride ions could induce excessive aggregation and precipitation of AgNPs, both are not preferred for SERS measurement. Moreover, the SERS spectra of analytes are time dependent between the induction of the aggregation and the measurement $[19,20]$. However, to our knowledge, there are no systematic or comprehensive studies that aim at evaluating the effect of $\mathrm{NaCl}$ concentrations and the time of incubating paper substrate in colloidal AgNPs to paper SERS performance.

Analysis of semen (or seminal plasma, SP) is important for forensic analysis to identify and differentiate semen from unknown fluids at sexual assault crime scenes [21] and it is also aimed at facilitating the examination and evaluation of male fertility $[22,23]$. However, at the laboratory level, these analyses require well-trained personnel, and the specimens must be subjected to pretreatment steps. In contrast, Raman spectroscopic methods have gained importance in the evaluation of semen samples because of their appeal in rapid, simple, and nondestructive analysis [21, 24, 25]. Obtaining high-quality and sensitive Raman spectra of SP while avoiding fluorescence interferences, especially those from large protein molecules in SP, is still a challenge. To the best of our knowledge, this is the first report conducting the potential detection and analysis of seminal plasma. Herein, SP was chosen as a practical detection target to evaluate and optimize the effect of $\mathrm{NaCl}$ concentration and soaking time with colloidal AgNPs to the performance of paper SERS substrate, and the preliminary study was conducted for potential detection and diagnosis of SP related diseases based on SP SERS analysis.

\section{Materials and Methods}

Whatman Number 3 grade filter paper was purchased from Whatman International, Ltd. (Maidstone, England). Silver nitrate, sodium citrate, Rhodamine 6G (R6G), and sodium chloride were all purchased from Sigma-Aldrich and used without further purification. Ultrapure water purified with a Millipore system (Milli-Q, USA) was used for all aqueous solution preparation and rinsing procedures. Millipore PES filter membranes ( $33 \mathrm{~mm}$ diameter, $0.22 \mu \mathrm{m}$ pore size) were purchased from Merck Millipore.

Semen samples were obtained with informed consent from male subjects who visited Fujian Maternal and Child Health Hospital for routine fertility test. After liquefaction, semen samples were immediately transported to the laboratory. Spermatozoa were removed from liquefied semen samples by centrifugation at $3000 \mathrm{rpm}$ for 10 minutes in an Eppendorf centrifuge; a certain volume of SP was then loaded into a syringe attached to a Millipore PES filter to remove unwanted residual spermatozoa as well as white cells to obtain filtered SP samples.

Figure 1 shows the schematic diagram of paper SERS substrate preparation and the corresponding SERS detection of SP samples. For paper SERS substrate fabrication, colloidal AgNPs were prepared via reduction of $\mathrm{AgNO}_{3}$ by sodium citrate using the modified method of Lee and Meisel [26]. After that, colloidal AgNPs were centrifuged (5000 rpm, $10 \mathrm{~min}$ ), bringing it to 20 times its original concentration. Filter paper with the size of $0.5 \mathrm{~cm} \times 0.5 \mathrm{~cm}^{2}$ was firstly soaked in $\mathrm{NaCl}$ solution for $5 \mathrm{~min}$ and subsequently transferred to the Petri dish containing concentrated AgNPs for different periods of time (Figure 1(b)) to achieve various degrees of AgNPs adsorption on the paper substrate.

For SERS detection, $5 \mu \mathrm{L}$ of filtrated SP was pipetted onto paper SERS substrate, which was then moved to Renishaw micro-Raman system for SERS measurement (Figure 1(c)). Confocal micro-Raman system (Renishaw inVia, UK) equipped with $785 \mathrm{~nm}$ diode laser was employed for SERS measurement. A $20 \mathrm{x}$ objective (NA $=0.4$, Leica) was used to focus excitation light and collect the backscattered signals. The laser power was set at $\sim 3 \mathrm{~mW}$ for each SP sample measurement; SERS spectra were obtained from three different locations near the center of the sample spot. Each spectrum with a range of $600-1800 \mathrm{~cm}^{-1}$ was obtained with a typical exposure time of 10 seconds. The Renishaw WIRE 3.4 software was utilized for spectral acquisition and cosmic ray removal. Prior to measurement, the instrument was calibrated with a silicon standard whose Raman peak is centered at $520 \mathrm{~cm}^{-1}$.

\section{Results and Discussion}

Colloidal AgNPs were widely used for SERS application due to their high SERS performance and ease of fabrication. By contrast, paper SERS substrates fabricated via soaking method have not only demonstrated high enhancement factors [27-29], but also offer several distinctive features such as low cost, flexibility, and high surface area, making them an attractive platform for SERS. Moreover, powders and residues, which are incredibly difficult to detect with rigid substrates or microfluidic devices, can be loaded into the paper SERS device by swabbing the inherently flexible device across a wide-area surface of any topology. Aggregation of AgNPs is known to play a key role in intense SERS 


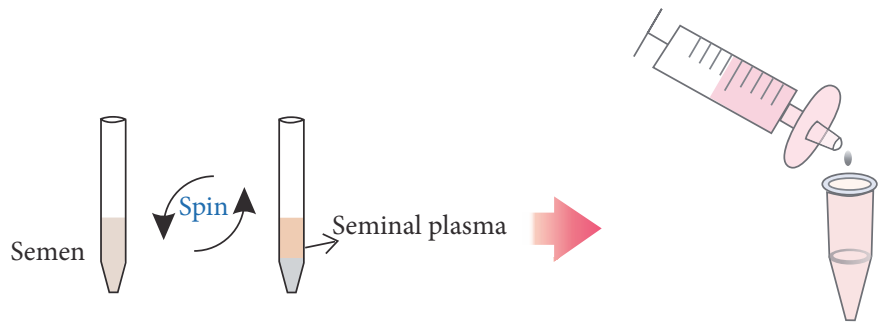

(a)

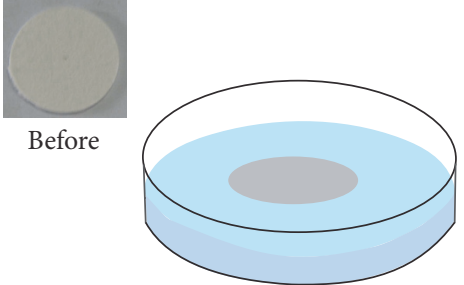

Filter paper soaked with $\mathrm{NaCl}$

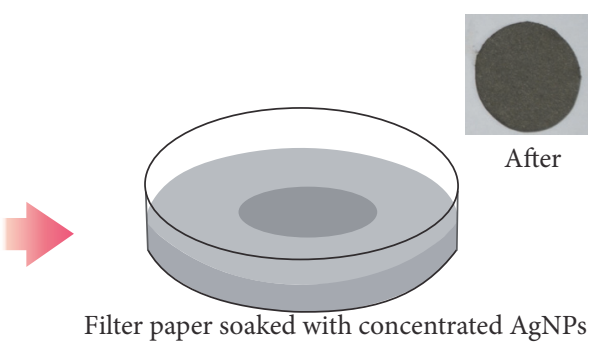

(b)

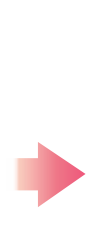

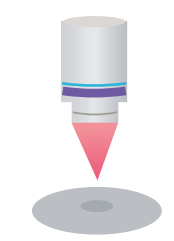

Sample measurement

Sample loading

(c)

FIGURE 1: Schematic illustration of SP sample and paper SERS substrate preparation as well as the corresponding detection of SP sample. Top to bottom: SP separation and filtration (a), filter paper soaked with $\mathrm{NaCl}$ solution and concentrated AgNPs solution (b), and SP sample loading and SERS measurement (c).

enhancement. Previous studies have demonstrated that aggregating agents such as $\mathrm{K}_{2} \mathrm{SO}_{4}, \mathrm{NaNO}_{3}$, and $\mathrm{NaCl}$ can produce an ultrahigh performance of signal enhancement by increasing the interactions between colloid nanoparticles to give rise to more hotspots [30], which in turn facilitate the preparation of simple, rapid, and high-performance SERS substrates. $\mathrm{NaCl}$ was specially selected due to its high performance as an ideal aggregating agent for citrate-reduced colloid [31, 32].

It has been reported that interference peaks may occur when the aggregating agent was added to colloidal nanoparticles [33]. Therefore, the SERS background signal as well as SERS spectra of SP from paper SERS substrate were obtained and thoroughly compared. As shown in Figure 2, SERS background from filter paper with or without $\mathrm{NaCl}$ treatment indicated neither Raman interferences nor any anomalous bands from the citrate-reduced silver colloid background. In contrast, it can be easily observed that SERS spectra of SP showed intense characteristic Raman bands once the SP was introduced. SERS spectra with high similarity in spectral profiles were obtained from paper substrates treated with or without $\mathrm{NaCl}$; however, the former demonstrated larger Raman peaks in the range from 600 to $1800 \mathrm{~cm}^{-1}$, especially the band region between 1100 and $1800 \mathrm{~cm}^{-1}$, from which the intensity of $1220 \mathrm{~cm}^{-1}$ peak was found to increase by 50 percent. According to the previous results [34], these major bands were mainly assigned to ascorbic acid $\left(1132 \mathrm{~cm}^{-1}, 1664 \mathrm{~cm}^{-1}\right)$, L-serine $\left(1220 \mathrm{~cm}^{-1}\right)$, nucleic acid, tryptophane $\left(1323 \mathrm{~cm}^{-1}\right)$, acetoacetate $\left(1445 \mathrm{~cm}^{-1}\right), \mathrm{L}-$ phenylalanine $\left(1584 \mathrm{~cm}^{-1}\right)$, and citric acid $\left(1695 \mathrm{~cm}^{-1}\right)$ as well. Peak shift was observed between 1400 and $1800 \mathrm{~cm}^{-1}$, exhibiting selective enhancement due to the fact that paper substrate has specific affinity to some molecule species.

Incubating filter paper with colloidal AgNPs for different lengths of time resulted in a clear and uniform color change of filter paper over a large length scale, which means a different amount of AgNPs was adsorbed onto the filter paper. To quantify the amount of AgNPs adsorbed onto the paper substrate, the color intensity of paper substrate was recorded and analyzed with ImageJ software (NIH, Maryland, USA). Figure 3 shows the relationship between the color intensity of paper substrate and soaking time with colloidal AgNPs under different $\mathrm{NaCl}$ concentrations. Under constant $\mathrm{NaCl}$ concentrations, the color of the paper substrate darkens with the increase of soaking time with colloidal AgNPs. Surprisingly, for the control group (without $\mathrm{NaCl}$ treatment), 


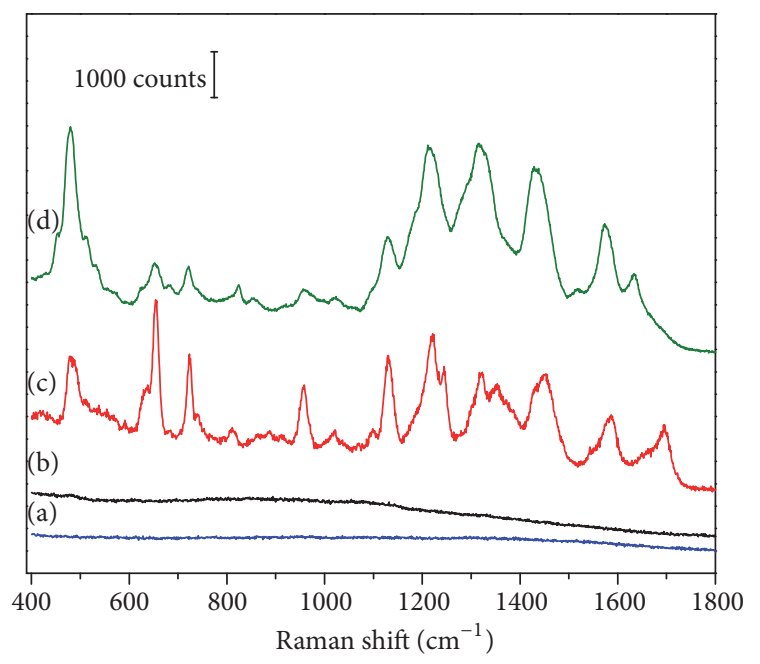

- SERS background without $\mathrm{NaCl}$
SERS background with $\mathrm{NaCl}$
- SP SERS without $\mathrm{NaCl}$
SP SERS with $\mathrm{NaCl}$

FIGURE 2: SERS background of paper substrate treated with (a) or without (b) $\mathrm{NaCl}$ solution, and SERS spectra of SP on paper substrate treated without (c) or with (d) $20 \mathrm{mM} \mathrm{NaCl}$ solution.

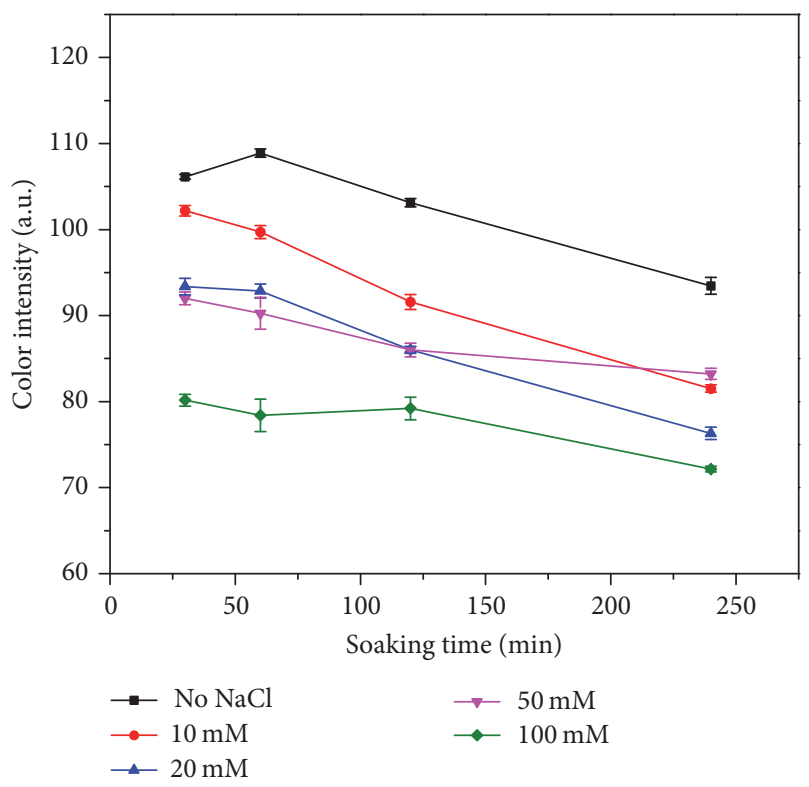

FIGURE 3: Color intensity of filter paper treated with AgNP solutions with different concentrations of $\mathrm{NaCl}$ at different soaking time.

the color intensity at $30 \mathrm{~min}$ soak was slightly lower than that at $1 \mathrm{~h}$ soak; however, the exact reason for this is unclear. In general, the higher the concentration of $\mathrm{NaCl}$, the lower color intensity of paper substrate at the same incubation time with colloidal AgNPs. This is mainly because the chloride ion has a stronger affinity to AgNPs than the citrate ion, thus replacing the citrate ion from the surface of AgNPs. AgNPs can, therefore, be easily adsorbed onto the filter paper though the strong affinity between chloride ion and AgNPs [17]. It is worth mentioning that compared with higher concentrations of $\mathrm{NaCl}(50 \mathrm{mM}$ and $100 \mathrm{mM})$, paper treated with lower concentrations of $\mathrm{NaCl}(10 \mathrm{mM}$ and $20 \mathrm{mM})$ resulted in a greater color change over soaking time. This is mainly because rapid adsorption of AgNPs on the paper substrate can be achieved in a short period of time due to the high concentration of chloride ions. However, the subsequent soaking with colloidal AgNPs will eventually lead to precipitation of aggregated AgNPs. This means that simply increasing the time of soaking filter paper in colloidal AgNPs after its treatment with high concentration of $\mathrm{NaCl}$ did not effectively increase the adsorption of AgNPs on the paper substrate.

In order to further understand the specific distribution of AgNPs onto the paper substrate following the soaking process with colloidal AgNPs, a series of corresponding SEM images (Figure 4) were performed to provide sufficient details to characterize and compare the distribution of AgNPs on paper substrate under different conditions (concentrations of $\mathrm{NaCl}$, and soaking time). It can be seen that the density of AgNPs on filter paper surface increases over soaking time. Higher concentrations of $\mathrm{NaCl}$ will definitely have a greater impact on aggregation of AgNPs, showing that AgNPs tend to aggregate and form clusters in a shorter time. Accordingly, the surface density of AgNPs was observed to be much higher on the paper substrate treated with high concentrations of $\mathrm{NaCl}(50 \mathrm{mM}$ and $100 \mathrm{mM})$ than low concentrations (10 $\mathrm{mM}$ and $20 \mathrm{mM})$, especially before the soaking time point of $2 \mathrm{~h}$. As mentioned above, after treatment with high concentration of $\mathrm{NaCl}$ solution, the excessive soaking time will inversely lead to precipitation of aggregated AgNPs. As a result, more effective aggregation of AgNPs over time was observed by the treatment at $20 \mathrm{mM}$ $\mathrm{NaCl}$ (see Figure s1 in Supplementary Material, available online at https://doi.org/10.1155/2017/4807064).

In most cases, high-sensitivity detection of analytes is fairly important and urgently required, especially for the trace level detection of interesting targets. The clustering of AgNPs is responsible for high SERS activity of the substrates. In order to select an optimal SERS substrate potentially suitable for rapid and reliable analysis of SP, the impact of soaking time with colloidal AgNPs as well as $\mathrm{NaCl}$ concentration for pretreatment to SERS performance of paper substrate was investigated. The characteristic SP SERS band $\left(1220 \mathrm{~cm}^{-1}\right)$ shown in Figure 2 was selected, as illustrated in Figure 5, when the concentration of $\mathrm{NaCl}$ is $10 \mathrm{mM}$ and $20 \mathrm{mM} ; 1220 \mathrm{~cm}^{-1}$ band intensity gradually increased over soaking time; however, for concentration of $50 \mathrm{mM}$, the $1220 \mathrm{~cm}^{-1}$ band intensity fluctuated; particularly when the concentration of $\mathrm{NaCl}$ was $100 \mathrm{mM}, 1220 \mathrm{~cm}^{-1}$ band intensity decreased over soaking time; the possible explanation for this phenomenon is that when filter paper was treated with low concentration of $\mathrm{NaCl}, \mathrm{AgNPs}$ slowly accumulated on the paper substrate as soaking time increased; however, for high concentration of $\mathrm{NaCl}$, the amount of chloride ion adsorbed on filter paper was relatively higher; therefore, theoretically there were more AgNPs adsorbed on paper substrate, but excessive amount of $\mathrm{NaCl}$ will eventually lead to creation of too large AgNP aggregates or precipitation in order to still support the optimal electromagnetic enhancement. That 

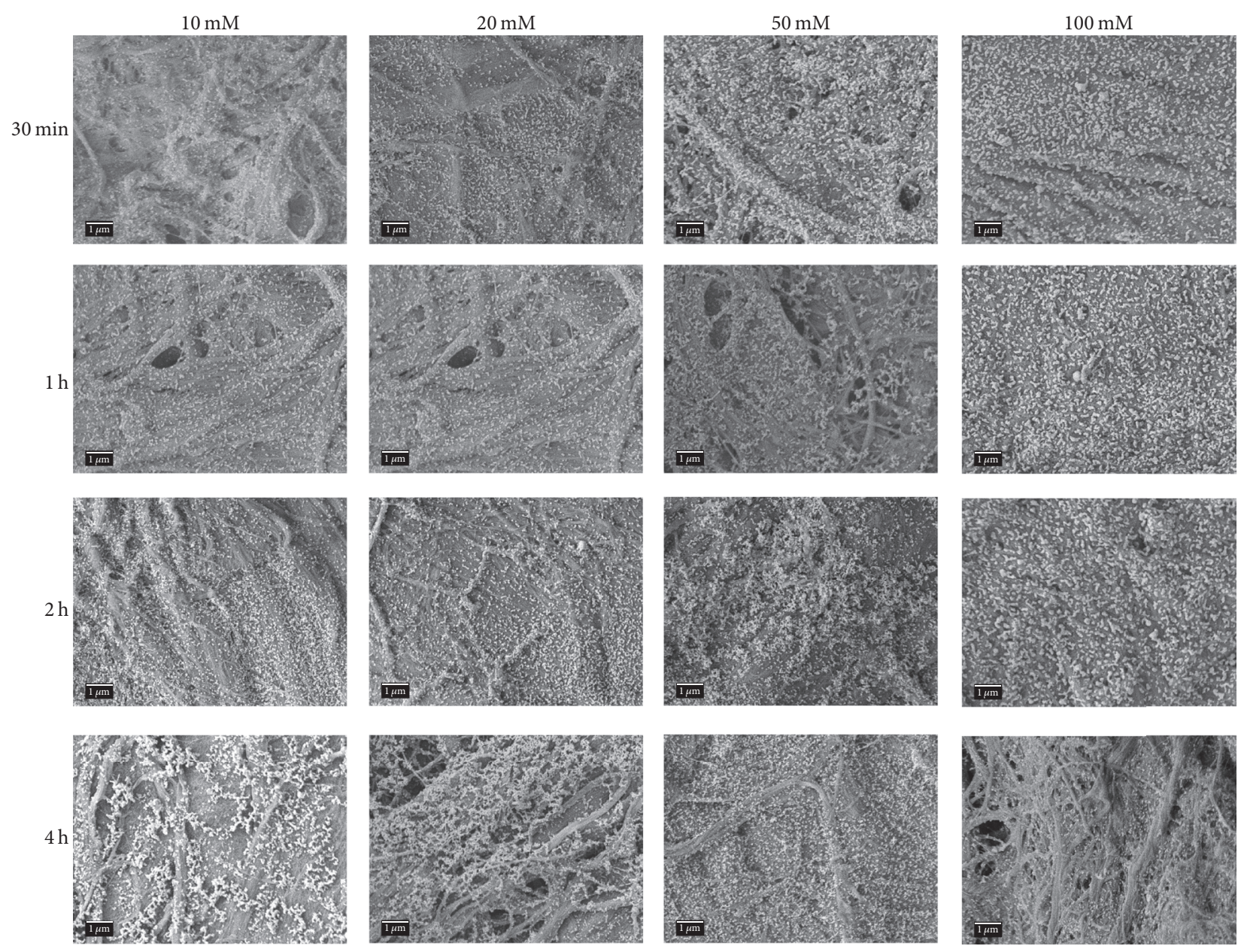

Figure 4: SEM images of AgNPs on filter papers treated with different concentrations of $\mathrm{NaCl}$ at different soaking time. The magnification is 5,000 times and the scale bar is $1 \mu \mathrm{m}$.

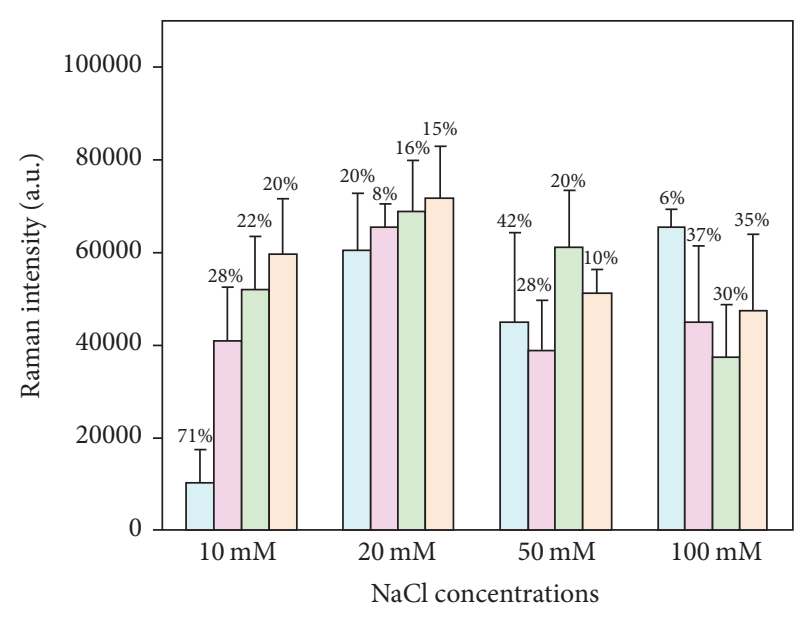

Soaking time

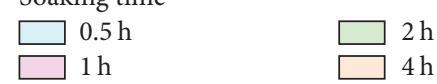

FIGURE 5: Comparison of $1220 \mathrm{~cm}^{-1}$ peak intensity of SP on paper substrate that soaked with colloidal AgNPs for different periods of time, under different $\mathrm{NaCl}$ concentrations. is, a low-density substrate may result in limited effective "hot spots"; however, high-density aggregates on the paper substrate may block the "hot spots." Meanwhile, the overall intensity of the SERS spectrum can be affected by competition between chloride ions and molecules from SP adsorbed at the surface of the AgNPs. Therefore, an optimal balance exists to achieve high-performance and reproducible SERS spectra.

An ideal SERS-active substrate requires a uniform distribution of nanoparticles to achieve reproducible measurements. To evaluate the reproducibility of SERS spectra of SP obtained from paper substrates under different conditions (see Figure s3), the relative standard deviation (RSD) of peak intensity at $1220 \mathrm{~cm}^{-1}$ was calculated accordingly (RSD values were given on top of each error bar in Figure 5). It can be easily found that when $\mathrm{NaCl}$ concentration is $10 \mathrm{mM}$, high RSD value (71\%) was observed at $0.5 \mathrm{~h}$ soak, but it decreased sharply as soaking time increased; this agreed well with the corresponding SEM images in Figure 4; for low concentration of $\mathrm{NaCl}$, AgNPs formed a sparse and uneven distribution, and fairly few clusters were observed; as soaking time increased, the AgNPs (or nanoclusters) were more uniformly distributed on paper surface. A similar 
situation occurred when treated with $50 \mathrm{mM} \mathrm{NaCl}$; more ordered and uniform AgNPs were shown, thus enabling not only high-performance SERS detection but also relatively lower RSD value. However, for $100 \mathrm{mM} \mathrm{NaCl}$, the minimum RSD value (6\%) was obtained at the beginning of $0.5 \mathrm{~h}$ soak; as soaking time increased the RSD value grew sharply and was maintained at a level between $30 \%$ and $40 \%$. The possible explanation is that for a high concentration of $\mathrm{NaCl}$, adsorption of AgNPs on paper advances quickly in a short time; as more and more AgNPs aggregated over time, precipitation happened, leading to an uneven distribution. In the meantime, excessive AgNPs loaded will form a relatively thick film layer [31], which in turn leads to a decrease in the numbers of SERS-active hot spots, thus eventually contributing to weak signal enhancement. In contrast, it can be seen that filter paper treated with $20 \mathrm{mM} \mathrm{NaCl}$ can bring AgNPs into optimally aggregated configuration; less soaking time will result in sparse adsorption of AgNPs on paper substrate; however, increasing the soaking time will increase the locally inhomogeneous adsorption of AgNPs; therefore, the most uniform and reproducible paper SERS substrate was achieved (RSD of $8 \%$ ) at incubation time of $1 \mathrm{~h}$ with colloidal AgNPs. Meanwhile, for the comparison of SERS spectra on paper substrate, SERS spectra of SP mixed with colloidal AgNPs for different periods of time, under different $\mathrm{NaCl}$ concentrations, were also analyzed (see Figure s2). Considerable similarities between them were observed. To be specific, the change of $1220 \mathrm{~cm}^{-1}$ peak intensity over time revealed that, in low $\mathrm{NaCl}$ concentrations (10 and $20 \mathrm{mM}$ ), signals increase slowly over time; however, in high $\mathrm{NaCl}$ concentration, the maximum intensity was achieved at $2 \mathrm{~h}$ and then decreased gradually. It can be found that the higher the $\mathrm{NaCl}$ concentration, the shorter the time it takes to achieve aggregation of AgNPs. Additional incubation time induces detrimental effect to SERS enhancement, owing to the precipitation of AgNPs clusters. Therefore, the optimal SERS results (especially the high reproducibility) was achieved under the treatment by $20 \mathrm{mM} \mathrm{NaCl}$, demonstrating similar optimization results achieved from the paper SERS substrate.

To take a close look at the performance of paper substrate treated by $20 \mathrm{mM} \mathrm{NaCl}$, Figure 6 shows the normalized mean SERS spectra of SP obtained from the paper substrate, which was soaked with colloidal AgNPs for different incubation periods. Apparently, all these SP SERS spectra presented very good signal-to-noise ratio and an abundance of intense SERS peaks, and these SERS spectra showed highly similar spectral profiles to each other, indicating excellent reliability as well as enhancement of paper substrate for SP SERS measurement. Moreover, the variations of SERS spectra recorded from 5 random positions of the paper substrate were low (shown in shaded part), showing excellent reproducibility.

To quantify the corresponding enhancing performance for the paper substrate which was achieved under abovementioned optimal conditions, Rhodamine 6G (R6G) was used to calculate the enhancement factor (EF), which was given by the following formula:

$$
\mathrm{EF}=\frac{I_{\mathrm{SERS}} / C_{\mathrm{SERS}}}{I_{\mathrm{RS}} / C_{\mathrm{RS}}},
$$

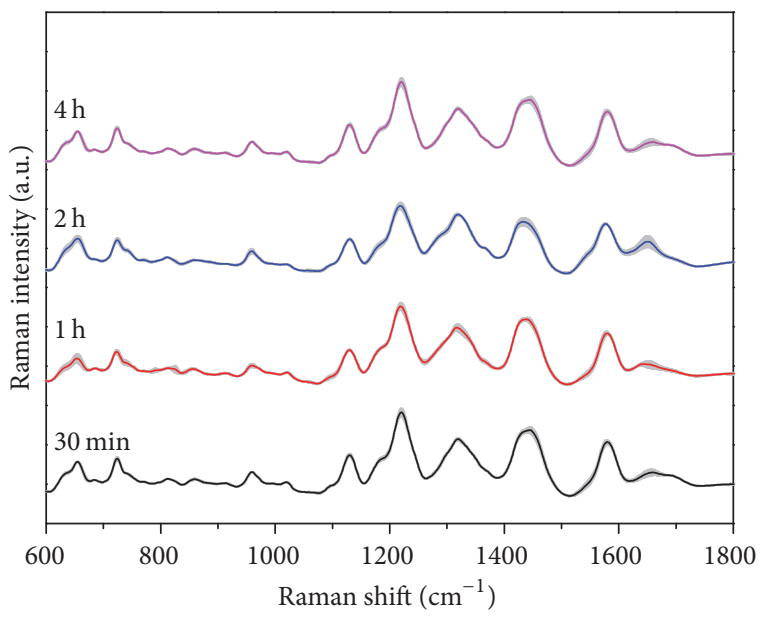

FIGURE 6: Mean SERS spectra of seminal plasma on paper substrate treated with $20 \mathrm{mM} \mathrm{NaCl}$ under different soaking time $(0.5 \mathrm{~h}, 1 \mathrm{~h}$, $2 \mathrm{~h}$, and $4 \mathrm{~h}$ ); shaded area indicates the standard deviations.

where $I_{\text {SERS }}$ and $I_{\mathrm{RS}}$ are the intensities of $1511 \mathrm{~cm}^{-1}$ of R6G for SERS and conventional Raman spectra (see Figure s4) and $C_{\text {SERS }}$ and $C_{R S}$ are the concentrations of R6G molecules in the scattering volume for SERS and Raman measurement. An average EF of $2.27 \times 10^{7}$ was achieved under this optimized condition.

Generally, sufficient loading of AgNPs on paper substrate requires relatively long soaking time (24 to 48 hours) [9, 15, 17]; this is mainly because filter paper is primarily composed of cellulose, which contains a large amount of negatively charged carboxyl groups $[35,36]$; therefore it is understandable that relatively long incubation period is needed to accumulate enough amount of AgNPs on paper substrate for optimal SERS performance; however, SERS activity of paper substrate has the risk of being influenced due to oxidation of AgNPs induced by long-term exposure to air or even in solution $[37,38]$ as well as the potential decay of filter paper after a long time soaking with AgNPs solution. By contrast, our proposed method does offer a simple and rapid way to prepare a highly sensitive paper SERS substrate, and the overall time needed can be significantly reduced to less than $2 \mathrm{~h}$. Increasing the concentration of AgNPs and/or aggregating agent of $\mathrm{NaCl}$ as well as accelerating the drying process for the paper substrate may further potentially help shorten the preparation time for SERS measurement.

On the other hand, it is worth mentioning that the laser spot size on paper substrate is merely tens of $\mu \mathrm{m}^{2}$ during measurement; however, the rough surface of filter paper and the difference in affinity to different molecules can cause analytes to diffuse and accumulate in different locations before detection. Therefore, it is reasonable that the small detection area may lead to changes of SERS spectral profile as well as peak intensities. Increasing excitation spot size (e.g., using fiber-based portable Raman system) would potentially ensure more reproducible and representative SERS spectra which are critical for rapid and on-site Raman analysis. 


\section{Conclusions}

In this work, we have demonstrated that for paper SERS substrate both $\mathrm{NaCl}$ concentration and the soaking time with colloidal AgNPs have a great impact on SERS performance, which was explained in detail with the corresponding SEM images of AgNPs on the paper substrate. An optimized condition $(20 \mathrm{mM} \mathrm{NaCl}$, $1 \mathrm{~h}$ soak with colloidal AgNPs) for achieving excellent EF of $2.27 \times 10^{7}$ was established. Importantly, clinical SP samples were preliminarily performed and evaluated, showing a reproducible SERS performance (RSD of $8 \%$ ). Pretreatment with $\mathrm{NaCl}$ will definitely facilitate fabricating the paper SERS substrate more rapidly, and the realization and optimization of the paper SERS substrate will open up new opportunities for SERS applications in reliable on-site detection of SP and clinical diagnosis of fertilityrelated diseases as well.

\section{Conflicts of Interest}

The authors declare that they have no conflicts of interest.

\section{Acknowledgments}

This work was supported by the National Natural Science Foundation of China (nos. 61308113, 61178090, and 11274065) and the outstanding youth program sponsored by Fujian Department of Education, and the project was also supported by the Program for Changjiang Scholars and Innovative Research Team in University (Grant no. IRT_15R10) and China Scholarship Council.

\section{References}

[1] E. C. Le Ru, E. Blackie, M. Meyer, and P. G. Etchegoint, "Surface enhanced raman scattering enhancement factors: a comprehensive study," The Journal of Physical Chemistry C, vol. 111, no. 37, pp. 13794-13803, 2007.

[2] K. Kneipp, H. Kneipp, R. Manoharan et al., "Extremely large enhancement factors in surface-enhanced Raman scattering for molecules on colloidal gold clusters," Applied Spectroscopy, vol. 52, no. 12, pp. 1493-1497, 1998.

[3] S. Nie and S. R. Emory, "Probing single molecules and single nanoparticles by surface-enhanced Raman scattering," Science, vol. 275, no. 5303, pp. 1102-1106, 1997.

[4] G. Herrera, A. Padilla, and S. Hernandez-Rivera, "Surface Enhanced Raman Scattering (SERS) studies of gold and silver nanoparticles prepared by laser ablation," Nanomaterials, vol. 3, no. 1, pp. 158-172, 2013.

[5] S. Schlücker, "Surface-enhanced Raman spectroscopy: concepts and chemical applications," Angewandte Chemie International Edition, vol. 53, no. 19, pp. 4756-4795, 2014.

[6] S. W. Lee, Y.-B. Shin, K. S. Jeon et al., "Electron beam lithography-assisted fabrication of Au nano-dot array as a substrate of a correlated AFM and confocal Raman spectroscopy," Ultramicroscopy, vol. 108, no. 10, pp. 1302-1306, 2008.

[7] R. Gupta and W. A. Weimer, "High enhancement factor gold films for surface enhanced Raman spectroscopy," Chemical Physics Letters, vol. 374, no. 3-4, pp. 302-306, 2003.
[8] J. F. Betz, W. W. Yu, Y. Cheng, I. M. White, and G. W. Rubloff, "Simple SERS substrates: Powerful, portable, and full of potential," Physical Chemistry Chemical Physics, vol. 16, no. 6, pp. 2224-2239, 2014.

[9] Y. H. Ngo, D. Li, G. P. Simon, and G. Garnier, "Gold nanoparticle-paper as a three-dimensional surface enhanced raman scattering substrate," Langmuir, vol. 28, no. 23, pp. 87828790, 2012.

[10] E. P. Hoppmann, W. W. Yu, and I. M. White, "Inkjet-printed fluidic paper devices for chemical and biological analytics using surface enhanced Raman spectroscopy," IEEE Journal on Selected Topics in Quantum Electronics, vol. 20, pp. 195-204, 2014.

[11] P. M. Fierro-Mercado and S. P. Hernández-Rivera, "Highly sensitive filter paper substrate for sers trace explosives detection," International Journal of Spectroscopy, vol. 2012, Article ID 716527, 7 pages, 2012.

[12] W. W. Yu and I. M. White, "Inkjet-printed paper-based SERS dipsticks and swabs for trace chemical detection," Analyst, vol. 138, no. 4, pp. 1020-1025, 2013.

[13] T. Vo-Dinh, M. Y. K. Hiromoto, G. M. Begun, and R. L. Moody, "Surface-enhanced raman spectrometry for trace organic analysis," Analytical Chemistry, vol. 56, no. 9, pp. 1667-1670, 1984.

[14] W. W. Yu and I. M. White, "Inkjet printed surface enhanced raman spectroscopy array on cellulose paper," Analytical Chemistry, vol. 82, no. 23, pp. 9626-9630, 2010.

[15] C. H. Lee, L. Tian, and S. Singamaneni, "Paper-based SERS swab for rapid trace detection on real-world surfaces," ACS Applied Materials and Interfaces, vol. 2, no. 12, pp. 3429-3435, 2010.

[16] C. Desmonda, S. Kar, and Y. Tai, "Formation of gold nanostructures on copier paper surface for cost effective SERS active substrate-Effect of halide additives," Applied Surface Science, vol. 367, pp. 362-369, 2016.

[17] W.-L.-J. Hasi, X. Lin, X.-T. Lou et al., "Chloride ion-assisted self-assembly of silver nanoparticles on filter paper as SERS substrate," Applied Physics A, vol. 118, no. 3, pp. 799-807, 2015.

[18] M. Çulha, M. Kahraman, N. Tokman, and G. Türkoğlu, "Surface-enhanced Raman scattering on aggregates of silver nanoparticles with definite size," Journal of Physical Chemistry C, vol. 112, no. 28, pp. 10338-10343, 2008.

[19] I. J. Hidi, M. Jahn, M. W. Pletz, K. Weber, D. Cialla-May, and J. Popp, "Toward levofloxacin monitoring in human urine samples by employing the LoC-SERS technique," Journal of Physical Chemistry C, vol. 120, no. 37, pp. 20613-20623, 2016.

[20] W. Shen, X. Lin, C. Jiang et al., "Reliable quantitative SERS analysis facilitated by core-shell nanoparticles with embedded internal standards," Angewandte Chemie-International Edition, vol. 54, no. 25, pp. 7308-7312, 2015.

[21] K. Virkler and I. K. Lednev, "Analysis of body fluids for forensic purposes: from laboratory testing to non-destructive rapid confirmatory identification at a crime scene," Forensic Science International, vol. 188, no. 1-3, pp. 1-17, 2009.

[22] D. R. Franken and S. Oehninger, "Semen analysis and sperm function testing," Asian Journal of Andrology, vol. 14, no. 1, pp. 6-13, 2012.

[23] C. Brazil, "Practical semen analysis: from A to Z," Asian Journal of Andrology, vol. 12, no. 1, pp. 14-20, 2010.

[24] K. Virkler and I. K. Lednev, "Raman spectroscopic signature of semen and its potential application to forensic body fluid identification," Forensic Science International, vol. 193, no. 1-3, pp. 56-62, 2009. 
[25] Z. Huang, X. Chen, Y. Chen et al., "Raman spectroscopic characterization and differentiation of seminal plasma," Journal of Biomedical Optics, vol. 16, no. 11, pp. 110501-1105013, 2011.

[26] P. C. Lee and D. Meisel, "Adsorption and surface-enhanced Raman of dyes on silver and gold sols," The Journal of Physical Chemistry, vol. 86, no. 17, pp. 3391-3395, 1982.

[27] L.-L. Qu, D.-W. Li, J.-Q. Xue, W.-L. Zhai, J. S. Fossey, and Y.T. Long, "Batch fabrication of disposable screen printed SERS arrays," Lab on a Chip, vol. 12, no. 5, pp. 876-881, 2012.

[28] C. H. Lee, M. E. Hankus, L. Tian, P. M. Pellegrino, and S. Singamaneni, "Highly sensitive surface enhanced raman scattering substrates based on filter paper loaded with plasmonic nanostructures," Analytical Chemistry, vol. 83, no. 23, pp. 89538958, 2011.

[29] Y. H. Ngo, D. Li, G. P. Simon, and G. Garnier, "Effect of cationic polyacrylamides on the aggregation and SERS performance of gold nanoparticles-treated paper," Journal of Colloid and Interface Science, vol. 392, no. 1, pp. 237-246, 2013.

[30] L.-J. Xu, C. Zong, X.-S. Zheng, P. Hu, J.-M. Feng, and B. Ren, "Label-free detection of native proteins by surface-enhanced Raman spectroscopy using iodide-modified nanoparticles," Analytical Chemistry, vol. 86, no. 4, pp. 2238-2245, 2014.

[31] W. W. Yu and I. M. White, "A simple filter-based approach to surface enhanced Raman spectroscopy for trace chemical detection," Analyst, vol. 137, no. 5, pp. 1168-1173, 2012.

[32] M. B. Ross, M. J. Ashley, A. L. Schmucker et al., "StructureFunction Relationships for Surface-Enhanced Raman Spectroscopy-Active Plasmonic Paper," Journal of Physical Chemistry C, vol. 120, no. 37, pp. 20789-20797, 2016.

[33] N. R. Yaffe and E. W. Blanch, "Effects and anomalies that can occur in SERS spectra of biological molecules when using a wide range of aggregating agents for hydroxylamine-reduced and citrate-reduced silver colloids," Vibrational Spectroscopy, vol. 48, no. 2, pp. 196-201, 2008.

[34] X. Chen, Z. Huang, S. Feng et al., "Analysis and differentiation of seminal plasma via polarized SERS spectroscopy," International Journal of Nanomedicine, vol. 7, pp. 6115-6121, 2012.

[35] M. A. S. A. Samir, F. Alloin, and A. Dufresne, "Review of recent research into cellulosic whiskers, their properties and their application in nanocomposite field," Biomacromolecules, vol. 6, no. 2, pp. 612-626, 2005.

[36] Y. Habibi, L. A. Lucia, and O. J. Rojas, "Cellulose nanocrystals: chemistry, self-assembly, and applications," Chemical Reviews, vol. 110, no. 6, pp. 3479-3500, 2010.

[37] M. Erol, Y. Han, S. K. Stanley, C. M. Stafford, H. Du, and S. Sukhishvili, "SERS not to be taken for granted in the presence of oxygen," Journal of the American Chemical Society, vol. 131, no. 22, pp. 7480-7481, 2009.

[38] R. Zhang, B.-B. Xu, X.-Q. Liu et al., "Highly efficient SERS test strips," Chemical Communications, vol. 48, no. 44, pp. 59135915, 2012. 

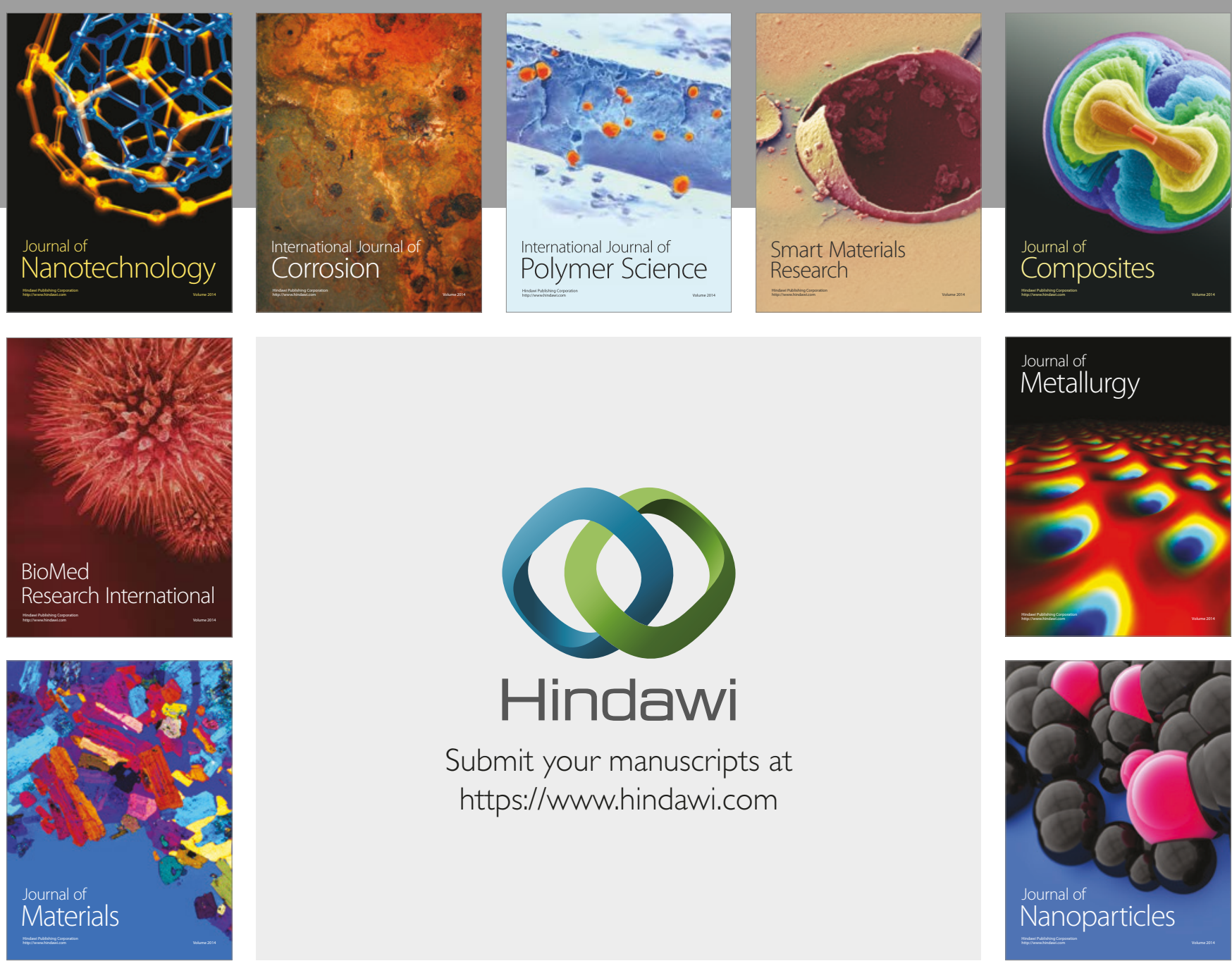

\section{Hindawi}

Submit your manuscripts at

https://www.hindawi.com
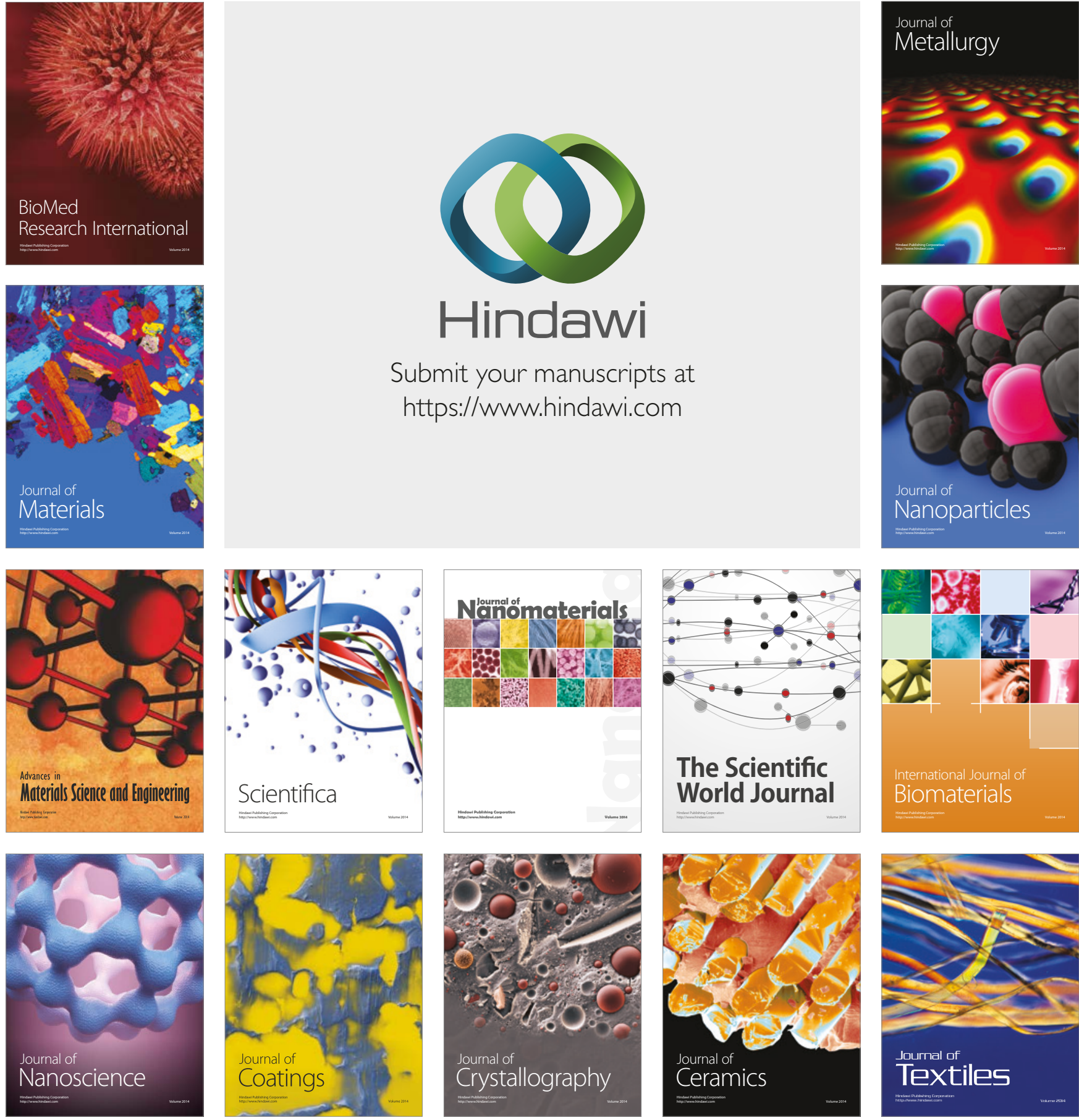

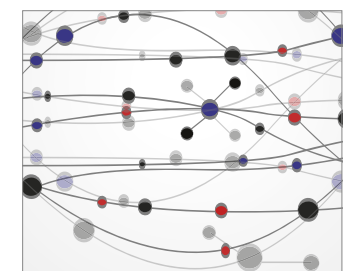

The Scientific World Journal
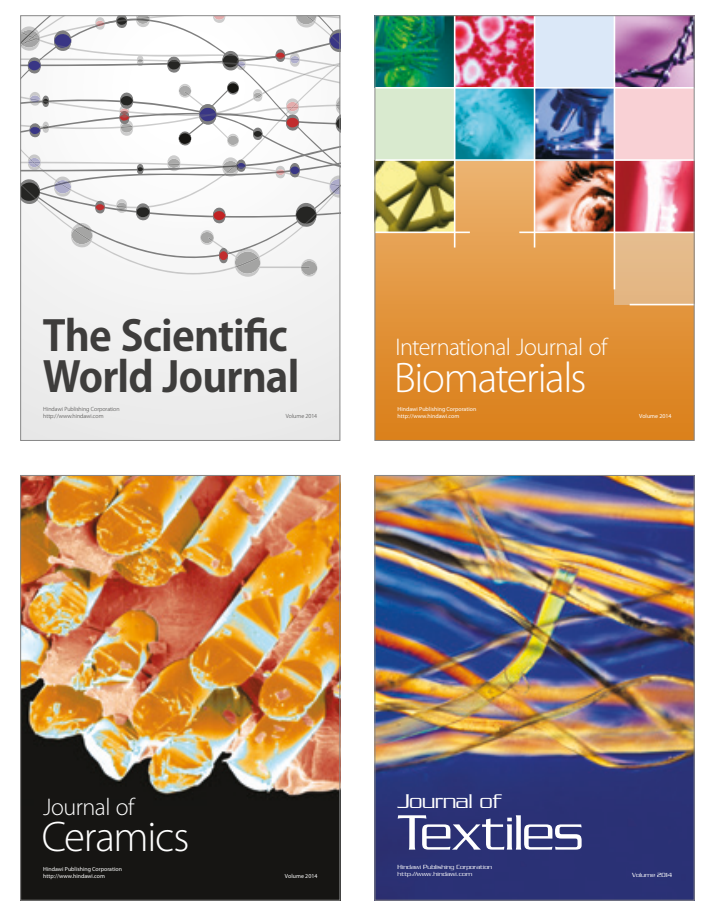